\title{
Effect of phosphorus dopant concentration on the carrier mobility in crystalline silicon
}

\author{
Yasab Ayele ${ }^{1}$, Zena Yohannes ${ }^{1}$ and Amare Benor ${ }^{1,2,3^{*}}$ \\ ${ }^{1}$ Department of Materials Science and Engineering, Bahir Dar University, Bahir Dar, P.O. Box. 79, Ethiopia \\ ${ }^{2}$ Department of Physics, Bahir Dar University, Bahir Dar, P.O. Box. 79, Ethiopia \\ ${ }^{3}$ Department of Physics, Addis Ababa University, P.O. Box 1176, Addis Ababa, Ethiopia
}

\begin{abstract}
This study investigated the effect of phosphorus dopant concentration on mobility of crystalline silicon (c-Si). It considers different temperature ranges, from $100 \mathrm{~K}$ to $500 \mathrm{~K}$, and dopant concentration from $10^{12} \mathrm{~cm}^{-3}$ to $10^{20}$ $\mathrm{cm}^{-3}$ in relation to its effect on the mobility of the crystalline silicon. This study indicates that the mobility of phosphorus doped silicon, n-type silicon, at different dopant concentration, tends to reduce as the temperature is increased. On the other hand, the mobility of the doped semiconductor, at different temperatures, showed different trends as the dopant concentration increases: I) mobility decreased in between $10^{15}$ to $10^{17} \mathrm{~cm}^{-3}$, II) mobility saturates doping concentrations less than $10^{14} \mathrm{~cm}^{-3}$, and III) mobility is not significantly affected, by increasing the temperature for high doping concentration $10^{18}$ to $10^{20} \mathrm{~cm}^{-3}$. The two issues, lattice and impurity, dominate one another depending on the doping concentration and temperature, and thus contributed to dependence of mobility on temperature, in different trend, while being dependent on the fundamental theory of doping in semiconductors. Based on the study, as the temperature gets higher for higher doping concentration, mobility by the impurity scattering increases while it decreases by the lattice scattering, the two cases balance one another, and as a result mobility becomes almost constant, that is, the rate of change of mobility is relatively insignificant.
\end{abstract}

Keywords: Semiconductor, Mobility, Lattice scattering, Impurity scattering

DOI: http://dx.doi.org/10.4314/ejst.v9i2.4

\section{INTRODUCTION}

Silicon is by far the dominant semiconductor material used in electronics and photonic devices. Since the birth of semiconductor industry, it has been the key semiconductor material, and it is seen as the backbone of electronics and photovoltaic industry (Lukasiak and Jakubowski, 2010). Its application in electronic devices, mainly in transistor, as well as in photonic and photovoltaic materials has entirely revolutionized our life style (Seto, 1985; Fourmond et al., 2011). In relation to its application, the electronic property is deeply investigated in many studies, where many of the studies are based on doping. The studies has made significant progress in detailed understanding of the material and played a key role in advanced device application. The studies in electronic property of the material are mainly based on pand n- type doped crystalline silicon, and it mainly focuses on conductivity/resistivity, mobility and related parameters. The mobility can refer the majority and/or the minority carrier mobility, that is, electron and hole mobility (Cardona, 2010; 2011). The carrier mobility is affected by scattering, and theoretically the scattering can be of the following type: i) phonon (lattice) scattering, ii) ionized impurity scattering, iii) scattering by neutral impurity atoms and defects, iv) carriercarrier scattering, and (v) piezoelectric scattering (Pierret, 2003). Of these, the charge carrier mobility is dominantly affected by the lattice scattering and impurity scattering (Bulusu and

\footnotetext{
*Corresponding author: amarebenor@yahoo.com

(C) This is an Open Access article distributed under the terms of the Creative Commons Attribution License (http://creativecommons.org/licenses/CC BY4.0).
} 
Walker, 2008). The lattice scattering is dominant at high temperature; and as a result the mobility decreases with increasing temperature. However, impurity scattering results when a charge carrier travels past an ionized dopant concentration. While all other parameters kept constant, the charge carrier mobility significantly affects performance of electronic devices (Fujii et al., 2014). Apparently, a higher mobility results in better device performance (Chan,1994; Yacobi, 2003; Doering and Nishi, 2014). As a result, mobility is particularly a key factor in the performance of electronic devices (Watanabe, 1999). The mobility of semiconductors has got scientific attention in relation to its technological impact. Considerable charge carrier mobility investigation has been made in earlier studies. Li et al. (1977) made a theoretical model on the dopant density and temperature dependence of electron mobility and resistivity in n-type silicon. His theory yields a modified Brooks-Herring formulation (Arora et al., 1982) which takes into account the anisotropic scattering effects and the impurity scattering mobility, at a given temperature $T$, is given by:

$\mu_{I}=\frac{7.3 \times 10^{17} T^{\frac{8}{2}}}{N_{I} G(b)}$

where $N_{I}$ is the number of ionized impurity atoms and $G(b)$ is a function given by:

$G(b)=\ln (b+1)-\frac{b}{b+1}$

where $b$ is given by:

$b=\frac{1.52 \times 10^{15} T^{2}}{n^{\prime}}$

where $n^{\prime}=n[2-(n / N)]$ and it assumes the acceptor concentration to be zero, $\mathrm{n}$ being electron density per cubic centimeter. Besides, a detailed review work was made on the charge transport properties of silicon (Jacoboni et al., 1977). Latter, Arora et al. (1982) derived analytical relation on the electron and hole mobility in silicon as a function of concentration and temperature. In his study, he noted that the lattice scattering mobility can be fitted very well by the following equation:

$\mu_{L}=8.56 \times 10^{8} T^{-2.33}$

Thus, after taking into account both effects $\mu_{\Lambda}$ and $\mu_{l}$, the total charge carrier mobility can be calculated. These former studies made significant contribution to the charge carrier transport. However, the very depth insight of charge transport is still somehow not well explained and theoretical modeling has been improved in time so as to match with the experimental results, as an example, Klassen mobility modeling. In particular, this modeling is not yet fully used in detailed study of carrier mobility of phosphorus doped silicon. Thus, the effect of dopant concentration, to make p- or n-type $\mathrm{Si}$, on the mobility of crystalline silicon is one key issue; and the case with a phosphorus dopant, Klassen mobility modeling, is our scientific issue. Consequently, we focus our attention on the characteristics of semiconductor materials that can be altered significantly by the addition of the impurity or doping into the crystalline silicon, which is an n-type c-Si doped by phosphorus. In other words, the study intends on extended insight of the effect of phosphorus dopant concentration on the carrier mobility of crystalline silicon. The study will see the effect of temperature and doping concentration on the mobility of the n-type semiconductor. Here, the study will consider the combined effect of both temperature and doping concentration and related trend in the electronic property of the material. Additionally, the two important related factors affecting the mobility, lattice and impurity scattering, will be discussed in detail in relation to the temperature and doping concentration. The study gen- 
erally indicates that lattice and impurity scattering dominate one another, in different rends, depending on the doping concentration and the temperature; and such a trend is well investigated in this study.

\section{METHODS}

This study examines the effect of phosphorus dopant concentration and temperature on charge carrier mobility of crystalline silicon (c-Si). The two most important conditions to vary in the study are temperature and dopant concentration. The temperature ranges from $100 \mathrm{~K}$ to $500 \mathrm{~K}$ while the dopant concentration ranges from $10^{12} \mathrm{~cm}^{-3}$ to $10^{20} \mathrm{~cm}^{-3}$. Consequently, the dependence of charge carrier mobility on temperature and dopant concentration is studied. The study used simulation based method on phosphorus doped crystalline silicon, where it uses existing software from the PV Light House, using Klassen 1992 mobility modeling, to generate data. The software is the tool to calculate mobility of crystalline silicon as a function of dopant concentration from $10^{12} \mathrm{~cm}^{-3}$ to $10^{20} \mathrm{~cm}^{-3}$ and temperature from $100 \mathrm{~K}$ to $500 \mathrm{~K}$ by 50 intervals. Finally, the row data, obtained from the simulation work or PV light house, is organized and plotted.

\section{RESULTS AND DISCUSSION}

Figure 1, shows the electron and hole mobility as a function of dopant concentration, with a range of $10^{12} \mathrm{~cm}^{-3}$ to $10^{20} \mathrm{~cm}^{-3}$, for phosphorus doped crystalline silicon at room temperature or $300 \mathrm{~K}$. As seen from the figure, the charge carrier mobility of holes and electrons tends to decline as the doping concentration increases. Besides, the charge carrier motilities, of electrons and holes, tend to be constant below $10^{15} \mathrm{~cm}^{-3}$ and above $10^{19} \mathrm{~cm}^{-3}$ dopant concentrations. Furthermore, it is evident that the carrier mobility of electrons is higher than that of holes. In intrinsic crystalline semiconductor, e.g., crystalline silicon, the only factor that affects mobility is the temperature or phonon effect, where the mobility decline as the temperature rises. However, in extrinsic semiconductor, like phosphorus doped semiconductor $\mathrm{c}-\mathrm{Si}$, there are two factors or two contributions affecting the charge carrier mobility, namely impurity scattering and lattice scattering (Beadle et al., 1995).

Thus, Phosphorus doped semiconductor c-Si, mobility is affected by these two factors: lattice and impurity scattering. As shown in Figure 1, the charge carrier mobility of electrons exceeds that of holes is due to the fact that the effective mass, in c-Si, of electrons is less than that of holes. Besides, the decline of charge carrier mobility, by increasing the doping concentration, appears to be related to the increased impurity scattering where it induce more carrier or electron-electron interaction and tends to reduce the charge carrier mobility. The mobility of electrons is about three times greater than that of holes for low doping concentration, e.g., $10^{16} \mathrm{~cm}^{-3}$, while it is less than two fold at higher concentration after $10^{19} \mathrm{~cm}^{-3}$.

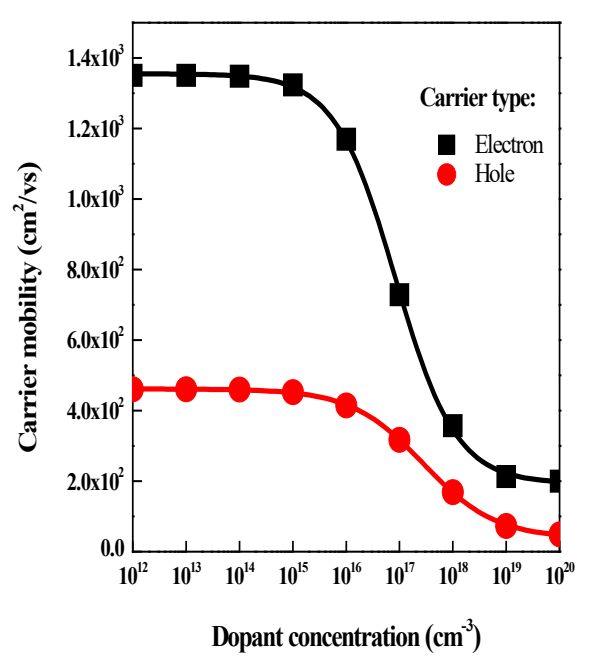

Figure 1. Charge carrier mobility, electrons and holes, as a function of dopant concentration of phosphors doped silicon at 300k. 
For doping concentration less than $10^{15} \mathrm{~cm}^{-3}$, the mobility of electron and hole is almost constant. In other words, as the doping concentration decreases from $10^{19} \mathrm{~cm}^{-3}$, carrier mobility is first insignificant, then shows a massive change with short ranges and finally saturates at low doping concentrations. The result also showed us that doping concentration around $10^{15} \mathrm{~cm}^{-3}$ is the threshold concentration to change from mobility saturation to mobility decrements at $300 \mathrm{~K}$.

At room temperature or $300 \mathrm{~K}$, mobility in a doped semiconductor is constant for low impurity concentrations because it is limited by the lattice scattering (Jeffrey, 2014). Thus, the study is consistent to former findings (Jacoboni et al., 1977). For impurity densities greater than $10^{15} \mathrm{~cm}$ 3 the mobility decreases as a result of impurity scattering. The significant difference in mobility might originate from being phonon scattering domination, in less doping concentration, to both lattice and majorly impurity scattering damnation at higher doping concentration.

To get deep insight on the effects of temprature on the charge carrier mobility, extended study was made at different temperatures from $100 \mathrm{~K}$ to $500 \mathrm{~K}$ with an interval of $50 \mathrm{~K}$. Figure 2 presents electron mobility as a function of phosphorus dopant concentration at different temperatures. At a given dopant concentration, the electron mobility tends to decrease as the temperature increases (Figure 2). The declining of the charge carrier mobility by the rise of temperature is attributed to the increased phonon/lattice scattering by the rise of the temperature. Impurity scattering is a major limiting factor at lower temperature while both impurity and lattice scattering is a limiting factor at higher temperature. The charge carrier mobility, at each temperature case, tends to saturate as the doping concentration declines, particularly

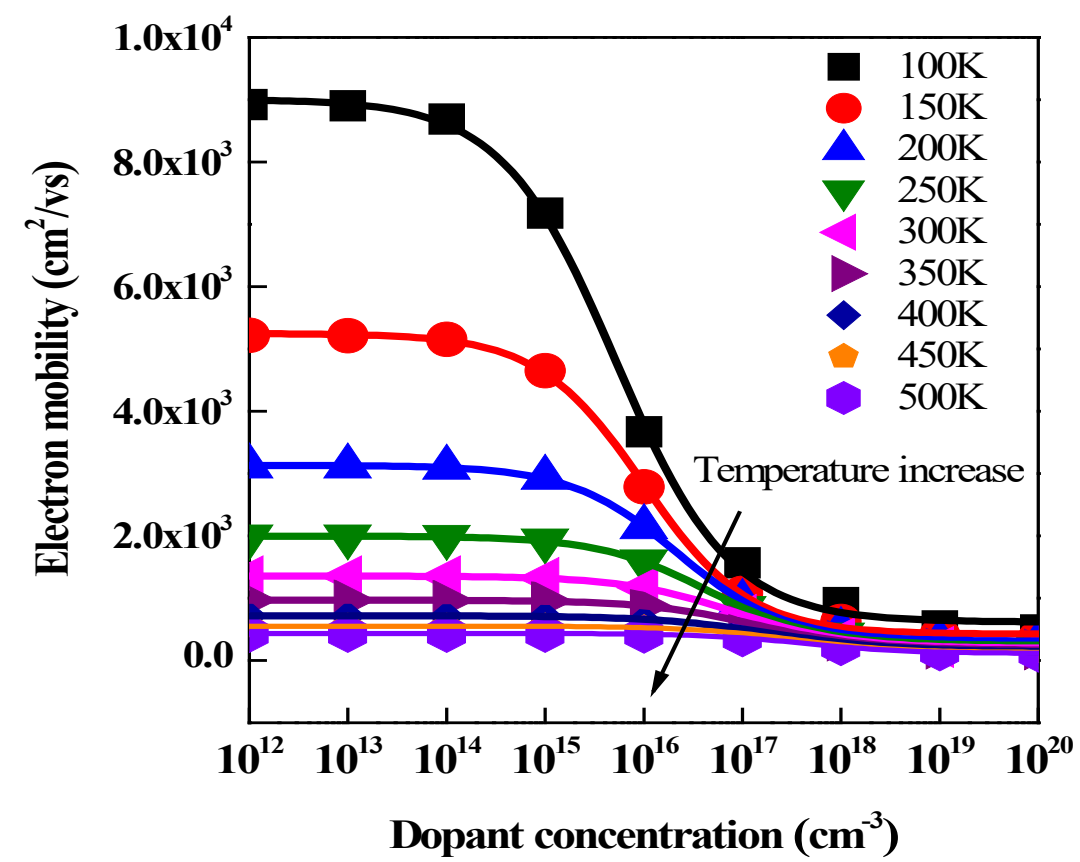

Figure 2. Electron mobility as a function of dopant concentration, of phosphors doped silicon, at different temperature (from $100 \mathrm{k}$ to $500 \mathrm{k}$ ). 
below doping concentration of $10^{15} \mathrm{~cm}^{-3}$ (Figure 1). Besides, it is evident that the charge carrier mobility is affected by the doping concentration. However, the case can be seen in three different cases i.e. the relatively high carrier mobility, low carrier mobility and the transition between the two cases. The mobility of the doped semiconductor, at different temperature, showed different three trends as the dopant concentration increases: I) mobility decreased in between $10^{15}$ to $10^{17} \mathrm{~cm}^{-3}$, II) mobility saturates for doping concentrations less than $10^{14} \mathrm{~cm}^{-3}$, and III) mobility is not significantly affected, almost constant, for high doping concentration greater than $10^{18} \mathrm{~cm}^{-3}$.

Here, the two factors, lattice and impurity scattering, dominate one another depending on the doping concentration and temperature, and thus contributed to dependence of mobility, in different trend, while being dependent on the fundamental theory of doping in semiconductors. For low dopant concentrations, less than $10^{16} \mathrm{~cm}^{-}$ ${ }^{3}$, the electron mobility declines by temperature or phonon/lattice scattering, and the lattice scattering has dominant effect particularly at low temperature. The relatively high carrier mobility, in the first regime, or concentrations less than $10^{15} \mathrm{~cm}^{-3}$ and mainly for low temperatures, is originated by the relatively low doping concentration. However, in the same regime and at the same doping concentration, the decrease of mobility by increasing the temperature is due to the increase of lattice scattering while the impurity scattering is relatively the same. Besides, as the temperature gets higher for higher doping concentration, mobility by the impurity scattering increases while it decreases by the lattice scattering, the two cases balance one another, and as result mobility as a function of temperature becomes relatively constant and the rate of change of mobility is relatively insignificant (Figure 3). Additionally, for a doping concentration less than $10^{15} \mathrm{~cm}^{-3}$ the mobility of electron is almost constant and is primarily limited by phonon scattering, that is, the rate of change of electron mobility is insignificant (Figure 3). However, for higher doping concentration, above $10^{17} \mathrm{~cm}^{-3}$, the carrier mobility is dominantly hindered by the impurity scattering and electron mobility is significantly influenced even for the cases with low temperature.

Here, it is important to note that the very high doping concentration, dominated by impurity scattering, the semiconductor almost reaches to its lowest mobility ranges and it attains to a phase that temperature no more bring a relatively significant change in charge carrier mobility, and thus mobility seems to be constant. The effect of temperature on carrier mobility is insignificant at higher doping concentration, and correspondingly the effect of doping concentration is also insignificant at higher temperature. Generally, as the temperature gets higher, the electron mobility decrease, since lattice vibration increases with increasing temperature,

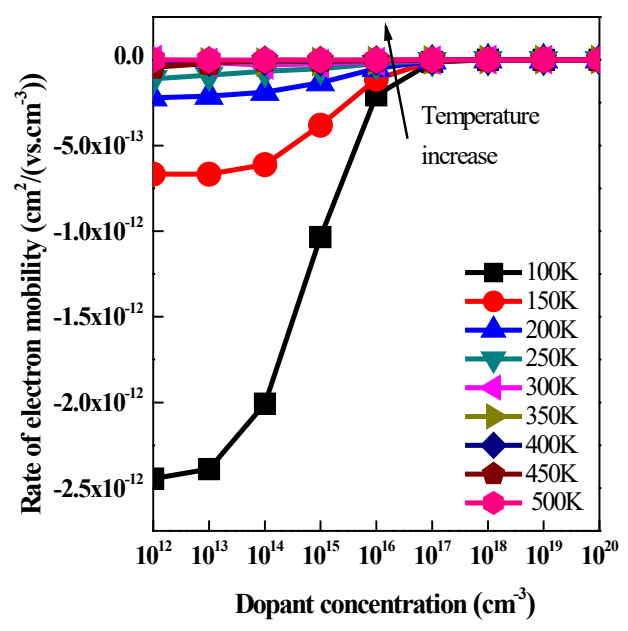

Figure 3. Rate of change of electron mobility, of phosphors doped silicon, as a function of dopant concentration at different temperature (from 100 $\mathrm{k}$ to $500 \mathrm{k}$ ). 
that is, lattice scattering becomes dominant at high temperatures; hence the mobility decreases with increasing temperature (Balasubramanian and Ariffin, 2014). The mobility of the carriers in a semiconductor is also influenced by the presence of charged impurities proportion to $\mathrm{T}^{3 / 2}$ (Tsao and Sah, 1976). At lower temperatures, impurity scattering dominates and it is governed as $\mathrm{T}^{-3 / 2}$ (Masettiet al., 1983). As a result, as the temperature increases, impurity scattering increases and the mobility decreases.

To get further uderstanding, the electron mobility as a function of temperature was made at different doping concentration ranging from $10^{12} \mathrm{~cm}^{-3}$ to $10^{20}$ $\mathrm{cm}^{-3}$. Correspondingly, the temperature range was made from $100 \mathrm{~K}$ to $500 \mathrm{~K}$. At a given temperature, the electron mobility tends to decreases as the doping concentration increases (Figure 4). This effect is particularly noticeable at low temperature, mainly for a temperature less than $300 \mathrm{~K}$. However, at higher temperatures, greater than $350 \mathrm{~K}$, the effect of concentration on the charge carrier mobility is insignificant.

The phenomena, mentioned above, witnessed the fact that the effect of doping concentration on carrier mobility is relatively insignificant at higher temperature and it is in consistent with the finding in Figure 2. It is evident that lattice/phonon and impurity scattering is dominating somehow above and below $400 \mathrm{~K}$, respectively. In the first case, below $350 \mathrm{~K}$, the decrease of the charge carrier mobility by the increasing of dopant concentration arises because of increased charged impurities that hinder the carrier mobility. The second case, above $350 \mathrm{~K}$, the mobility is already hindered by the high temperature and dopant concentration is insignificant, that is, lattice scattering dominates at higher temperature. Furthermore, the rate of change

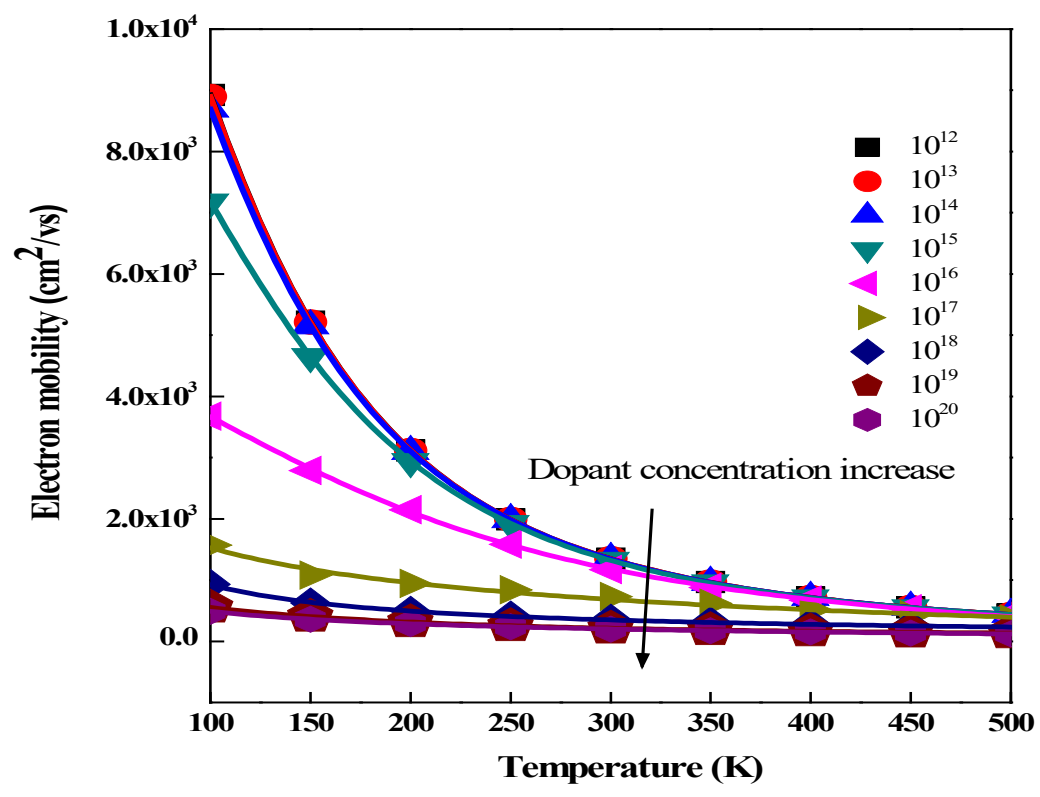

Figure 4. Electron mobility as a function temperature, of phosphors doped silicon, at different dopant concentration (from $10^{12} \mathrm{~cm}^{-3}$ to $10^{20} \mathrm{~cm}^{-3}$ ). 


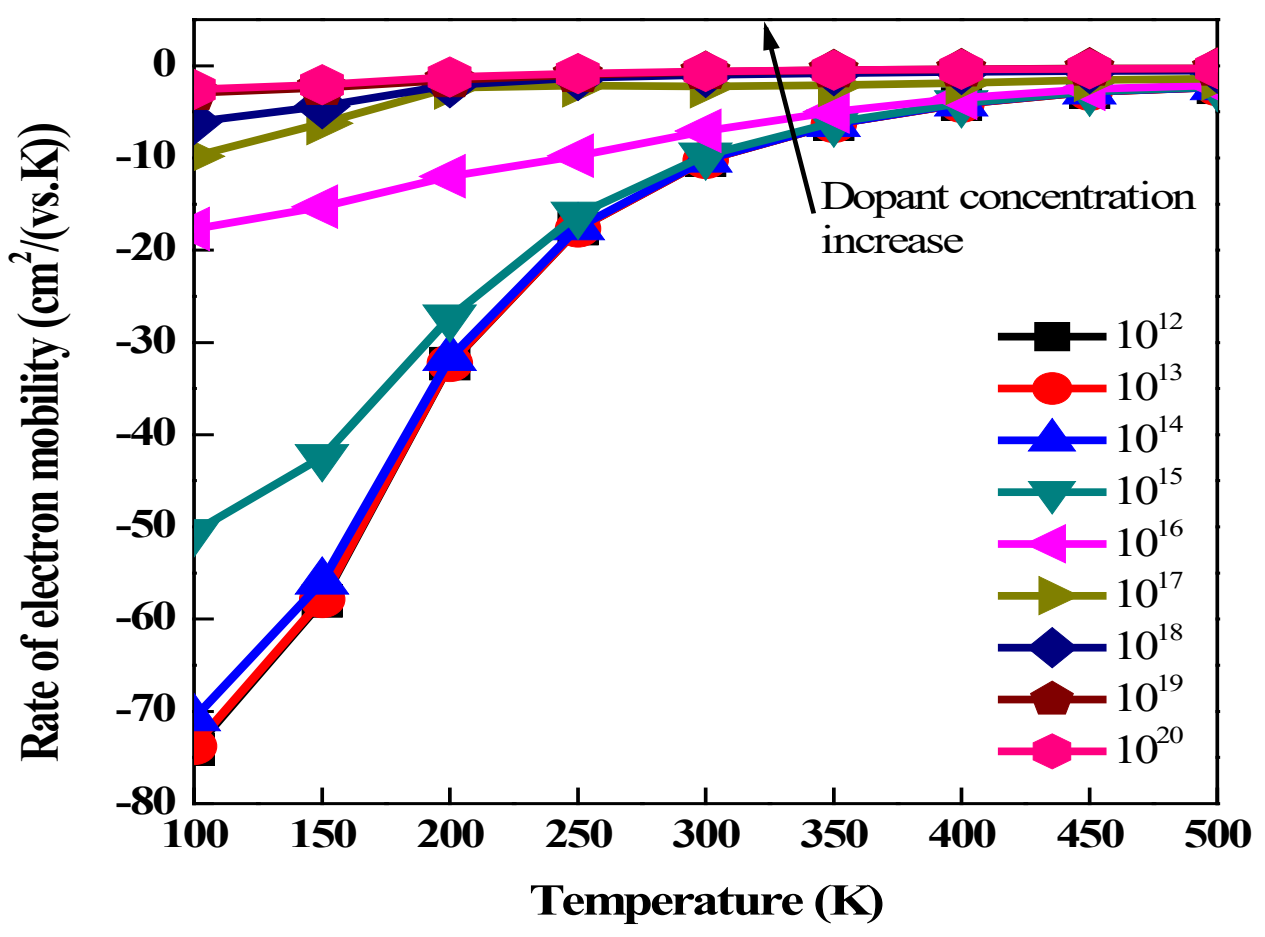

Figure 5. Rate of change of electron mobility, of phosphors doped silicon, as a function temperature at different dopant concentration (from $10^{12} \mathrm{~cm}^{-3}$ to $10^{20} \mathrm{~cm}^{-3}$ ).

of electron mobility as a function temperature is studied and presented in Figure 5. The rate of change of electron mobility, in temperature ranges, is relatively constant at high doping concentration, e.g., at doping concentration $10^{19} \mathrm{~cm}^{-3}$ and $10^{20}$ $\mathrm{cm}^{-3}$. Change in carrier mobility by increasing temperature is relatively insignificant. This effect, at high doping concentration, is attributed to the fact that the mobility is majorly dominated by impurity scattering and temperature has relatively insignificant contribution in charge carrier mobility. However, the effect is not the same at low doping concentrations, mainly for doping concentration less than $10^{16} \mathrm{~cm}^{-3}$. In this case, the rate of change of electron mobility, as a function of temperature, swiftly changes and it is related to its relatively low doping concentration and temperature being the dominant factor in charge carrier mobility. On the other hand, the rate of change of electron mobility is insignificant at higher temperature, particularly at high doping concentration, which tends to indicate that the semiconductor already reached to its high level of phonon/lattice scattering and the contribution of impurity is relatively insignificant that is, rate of change of mobility is insignificant maily at higher doping concentration.

\section{CONCLUSION}

In this study, we investigated that the temperature dependence of electron mobility, for phosphorus doped crystalline silicon, is dependent on impurity and lattice scattering with different trends depending on the doping concentrations. In general, when the doping concentration decreases from $10^{19} \mathrm{~cm}^{-3}$, carrier mobility is first insignificant, then shows a 
massive change with short ranges and finally saturates at low doping concentrations. The mobility of crystalline silicon decreases as dopant concentration increases, and this effect is pronounced at low temperature, particularly at $100 \mathrm{~K}$. Furthermore, as the temperature increases the carrier mobility decreases and such a trend is more observable at low doping concentration, particularly at $10^{12} \mathrm{~cm}^{-3}$. The study generally indicates that lattice and impurity scattering dominate one another, with different trends, depending on the doping concentration and the temperature.

\section{ACKNOWLEDGEMENTS}

The authors are grateful to $P V$ light house for the online simulation offer which helped us to systematically generate the data. Besides, the authors would like to acknowledge the International Science Program (ISP) for the grant support in relation to the Polymer Lab, in Physics Department, at Addis Ababa University (AAU).

\section{REFERENCES}

Arora, N. D., Hauser, J. R and Roulston, D. J. (1982). Electron and hole mobilities in Silicon as a function of concentration and temperature. IEEE Transactions on Electron Devices 9: 292- 295.

Balasubramanian, B and Ariffin, A.M. (2014). Current state-of-the-art solar photovoltaic technologies. Australian Journal of Basic and Applied Sciences 8:455-468.

Beadle, W. F., Tsai, J.C and Plummer, R.D. (1995). Quick Reference Manual for Semiconductor Engineers.Wiley, New York.

Bulusu, A and Walker, D. G. (2008). Review of Electronic Transport Model for Thermoelectric Materials, Super Lattices and Microstructures. Springer, New York.

Cardona, P. Y. (2010). Fundamentals of Semiconductors, Graduate Texts in Physics, $4^{\text {th }}$ Edition. Springer-Verlag Berlin Heidelberg.

Cardona, P. Y. (2011). Fundamentals of Semiconductors: Physics and Materials Properties. Springer, New Work.

Chan, J. (1994). Four-Point Probe Manual: EECS 143 Micro Fabrication Technology. http://www.inst.eecs.berkeley.edu, (accessed Nov. 13, 2014).

Nishi, Y and Doering, R. (2014). Handbook of Semiconductor Manufacturing Technology, $2^{\text {nd }}$ edition. Eugeniu, Borodin.

Fourmond, E., Forster, M., Einhaus, R and Lauvray, H. (2011). Electrical Properties of Boron, Phosphorus and Gallium Doped Silicon. Freiburg, Germany.

Fujii, M., Sugimoto, H., Hasegawa, M and Imakita, K. (2014). Silicon nanocrystals with high boron and phosphorus concentration. Journal of Applied Physics 115: 84301-84309.

Day, T. W., Zeier, W. G., Brown, D. R., Melot, B. C and Snyder, G. J. (2014). Determining conductivity and mobility values of individual components in multiphase composite $\mathrm{Cu}_{1.97} \mathrm{Ag}_{0.03}$ Se. Applied Physics Letters 105:172103-172108.

Jacoboni , C., Canali, C., Ottaviani G and Quaranta, A. A. (1977). A review of some charge transport properties of silicon. Solid State Electronics 20:77-89. 
Li, S and Thurde, W. R. (1977). The dopant density and temperature dependence of electron mobility and resistivity in n-type silicon. Solid-state Electronics 20: 609-616.

Lukasiak, L and Jakubowski, A. (2010). History of Semiconductors. Journal of Telecommunication and Information Technology 3:245-249.

Masetti, G., Severi, M and Solmi, S. (1983). Modelling of carrier mobility against Ccarrier concentration in Arsenic, Phosphorous and Boron doped Silicon. IEEE Transactions Electronics Device 30:764-853.

Pierret, R. F. (2003). Advanced Semiconductor

Fundamentals, $2^{\text {nd }}$ edition. Pearson

Education, New Jersey.

Seto, Y. W. (1985). Electrical properties of polycrystalline Silicon films. Journal of Applied Physics 46:5247- 5254.

Tsao, K. Y and Sah, C. T. (1976).Temperature dependence of mobility and electron conductivity mobility in p-type Silicon. Solid State Electronics 19:949- 953.

Watanabe, H. (1999). Driving Factors and Breakthroughs for Higher Performance Semiconductor Devices, DTIC Document.

Yacobi, B. G. (2003). Semiconductor

Materials: An Introduction to Basic

Principles. Springer, New Work. 